\title{
NLRC5: a NOD-like receptor protein with many faces in immune regulation
}

\author{
Yue Zhao ${ }^{1}$, Feng Shao ${ }^{1}$ \\ ${ }^{I}$ National Institute of Biological Sciences, 7\# Science Park Rd, Zhongguancun Life Science Park, Beijing 102206, China \\ Cell Research (2012) 22:1099-1101. doi:10.1038/cr.2012.83; published online 22 May 2012
}

Intracellular NOD-like receptor (NLR) proteins are believed to play important roles in innate immune detection or immune regulation, but only a few NLR proteins are functionally defined. Recent intensive studies, largely in vitro, on NLRC5 reveal multiple and controversial roles of this NLR protein in signaling regulation of both innate and adaptive immune responses. Using independently generated NIrc5-deficient mice, two studies have now established the physiological function of NLRC5 in transcriptional control of MHC class I molecules despite that its role in innate immune signaling remains to be further clarified.

In innate immunity, engagement of microbial products or danger signals by cognate pattern recognition receptors (PRRs) activates the proinflammatory NF- $\mathrm{kB}$ signaling, type I/II interferon responses, or macrophage inflammasome pathway, leading to transcriptional induction and/or secretion of a plethora of important inflammatory cytokines. NLR proteins represent a family of cytosolic PRRs that features an N-terminal caspase recruitment domain (CARD) or pyrin domain, a central nucleotidebinding and oligomerization domain,

Correspondence: Feng Shao

Tel: +86-10-80728593; Fax: 86-10-80728046

E-mail: shaofeng@nibs.ac.cn and a C-terminal leucine-rich repeat (LRR) domain. With a few exceptions including class II transactivator (CIITA), the NLR family, containing 23 members in human and 34 in mice, is generally thought to sense microbial products or danger signals for inflammasome activation.

NLRC5 (NLR family, CARD domain containing 5) is the largest NLR family member, consisting of 1866 aa with C-terminal 27 LRRs. Sequence analysis suggests that NLRC5 is most similar to CIITA among NLR family members. CIITA transactivates MHC class II expression by associating with the MHC enhanceosome. CIITA can also upregulate MHC class I expression in vitro, but plays no such roles in vivo, suggesting other factors involved in MHC class I activation. Similarly to CIITA, GFP-tagged NLRC5 shuttles between the cytosol and the nucleus, and could transcriptionally induce the expression of MHC class I and related genes by binding to promoters of these genes when expressed in human lymphoid and epithelial cell lines [1]. However, it is not known whether NLRC5 executes the same function under in vivo conditions. By generating Nlrc $5^{-/}$mice, now Yao et al. [2] and Tong et al. [3] convincingly demonstrate that mRNA levels of MHC class I (H2D, H2K, $\mathrm{H} 2 \mathrm{M} 3$ and $\mathrm{H} 2 \mathrm{Q} 1$ ) and its related genes (B2M, LMP2 and TAP1) were markedly reduced in $\mathrm{CD}^{+} \mathrm{T}$ cells, $\mathrm{CD} 8^{+} \mathrm{T}$ cells,
B cells as well as spleen and thymus tissues derived from the knockout animals. Both studies also show that NLRC5 deficiency has no effects on MHC class II expression, indicating a specific role in transactivation of MHC class I. As MHC class I antigen presentation is crucial for $\mathrm{CD} 8^{+} \mathrm{T}$ cell development and activation during pathogen infection, Yao et al. [2] challenged $N \operatorname{lrc} 5^{-/}$mice with Listeria monocytogenes (LM) and observed a drastically decreased number of $\mathrm{CD} 8^{+} \mathrm{T}$ cells but a normal profile of $\mathrm{CD}^{+} \mathrm{T}$ cells in spleens and livers of the mice. Their further functional analyses indicate that MHC class I-mediated $\mathrm{CD}^{+} \mathrm{T}$ cell activation in $\mathrm{Nlrc}^{-/-}$mice was largely impaired in response to LM infection. Consequently, the knockout animals became more susceptible to LM infection. Subsequent experiments collectively establish a critical role of NLRC5 in regulating MHC class I-mediated antigen-specific $\mathrm{CD} 8^{+} \mathrm{T}$ cell activation, proliferation as well as cytotoxicity, highlighting the importance of NLRC5 in host defense against bacterial infection. The function of NLRC5 in regulating MHC class I are further substantiated by three other competing studies [4-6], one of which shows that NLCR5 interacts with the RFX transcription factor complex to transactivate the promoters of MHC class I genes [4].

Consistent with its role in immune regulation and host defense, NLRC5 
is expressed in a wide spectrum of cell types and tissues but with high abundance in immune-related cells and organs with mucosal surfaces. NLRC5 expression can be induced by toll-like receptor (TLR) ligands like LPS and poly(I:C), interferons, or viral infection, but the most prominent effect is observed with IFN- $\gamma$ [1, 7-10]. IFN- $\gamma$ is a potent stimulator of MHC class I expression, which requires NLRC5 expression [1]. As demonstrated in the Yao study, NLRC5 could also induce IFN- $\gamma$ production through MHC class I-mediated $\mathrm{CD} 8^{+} \mathrm{T}$ cell activation. This positive feedback loop likely ensures a maximal immune response for efficient killing of intracellular pathogens. Using macrophages derived from $M y d 88^{-/}$, Irak4 ${ }^{-/}$and Stat1 $1^{-/-}$mice, Tong et al. [3] show that LPS induction of NLRC5 expression is indeed a secondary effect of IFN- $\beta$ secretion and more importantly that both IFN- $\beta$ and $-\gamma$ induce NLRC5 expression through the Stat1 signaling pathway.

The role of NLRC5 in regulating innate immune signaling has been controversial; two studies reported that NLRC5 negatively regulates NF- $\mathrm{KB}$ and type I interferon signaling [7-8] while another two suggested a positive function in virus-induced type I interferon response [9-10]. Consistent with their previous observation, Tong et al. again observed enhanced NF- $\mathrm{KB}$ activation and production of proinflammatory cytokines (IL-6 and TNF $\alpha$ ) in Nlrc $5^{-/}$ mouse embryonic fibroblasts (MEFs) upon TLR activation. Similarly, type I interferon signaling and production of IFN- $\alpha / \beta$ were augmented in $N$ lrc $5^{-/-}$ MEFs infected with vesicular stomatitis virus (VSV). The same phenomena were also recorded in $\mathrm{Nlrc5}^{-/}$peritoneal macrophages but less significantly in bone marrow macrophages (BMMs). Accordingly, $\mathrm{Nlrc}^{-/}$MEF cells were found to be more resistant to VSV infection. The negative role of NLRC5 in innate immune signaling has been attributed to biochemical interaction of NLRC5 with the IKK complex and RIG-I/MDA5 [8]. Although these findings provide a plausible function for the more abundant cytoplasmic expression of NLRC5, inconsistent or somewhat contradictory results are reported in the two new studies and also the previous Nlrc $5^{--}$knockout paper [11]. Data from Kumal et al. [11] and also Tong et al. themselves reveal no roles of NLRC5 in TLR signaling in bone marrow-derived dendritic cells (BMDCs). Yao et al. [2] also found that expression of NF- $\mathrm{kB}$ dependent or type I interferon genes were not affected by NLRC5 ablation in TLR ligand-treated or LM-infected BMMs. Consistent with this line of data, NLRC5 deficiency could not effectively protect the mice from LPS-induced septic shock and VSV infection. Therefore, the role of NLRC5 in regulating NF- $\mathrm{KB}$ and type I interferon signaling seems to be highly cell type and context dependent. Additional studies are needed to clarify the discrepancies obtained by different investigators and further define the exact function of NLRC5 in innate immune regulation.

Several NLR proteins including NLRP3 and NLRC4 form inflammasome complexes for capase- 1 activation and IL-1 $\beta$ or IL-18 maturation. RNAi knockdown of NLRC5 could diminish NLRP3-mediated caspase-1 activation and IL- $1 \beta$ production in human monocytic cells [12]. NLRC5 was shown to directly bind to NLRP3, a scenario similar to NAIP-instructed NLRC4 inflammasome activation in response to bacterial flagellin and type III secretion apparatus [13-14]. However, such inflammasome functions of NLRC5 were not confirmed in the previously generated knockout mice [11]. The situation is now further complicated by new in vivo data from Yao et al. [2] that seems to support a role of NLRC5 in the inflammasome pathway. In BMMs derived from their $\mathrm{Nlrc}^{-/}$mice, reduced caspase- 1 activation and more significantly IL- $1 \beta$ production were noted upon stimulation with different
NLRP3 agonists. NLRC5 also contributed to LM-induced IL- $1 \beta$ secretion; decreased neutrophil recruitment and higher bacterial burdens were observed in the spleens and livers of LM-infected Nlrc $5^{-1-}$ mice. These data seem to argue a promoting effect of NLRC5 in NLRP3 inflammasome activation. However, the rationale for this intriguing model and also the exact role of NLRC 5 in NLRP3 inflammasome activation remain to be further investigated.

\section{References}

1 Meissner TB, Li A, Biswas A, et al. NLR family member NLRC5 is a transcriptional regulator of MHC class I genes. Proc Natl Acad Sci USA 2010; 107:13794-13799.

2 Yao Y, Wang Y, Chen F, et al. NLRC5 regulates $\mathrm{MHC}$ class I antigen presentation in host defense against intracellular pathogens. Cell Res 2012; 22:836847.

3 Tong Y, Cui J, Li Q, Zou J, Wang HY, Wang RF. Enhanced TLR-induced NFkappaB signaling and type I interferon responses in NLRC5 deficient mice. Cell Res 2012; 22: 822-835.

4 Meissner TB, Liu YJ, Lee KH, et al. NLRC5 cooperates with the RFX transcription factor complex to induce MHC class I gene expression. J Immunol 2012; 188:4951-4958.

5 Neerincx A, Rodriguez GM, Steimle V, Kufer TA. NLRC5 controls basal MHC class I gene expression in an $\mathrm{MHC}$ enhanceosome-dependent manner. $J$ Immunol 2012; 188:4940-4950.

6 Staehli F, Ludigs K, Heinz LX, et al. NLRC5 deficiency selectively impairs MHC class I- dependent lymphocyte killing by cytotoxic T cells. J Immunol 2012; 188:3820-3828.

7 Benko S, Magalhaes JG, Philpott DJ, Girardin SE. NLRC5 limits the activation of inflammatory pathways. $J$ Immunol 2010; 185:1681-1691.

8 Cui J, Zhu L, Xia X, et al. NLRC5 negatively regulates the NF-kappaB and type I interferon signaling pathways. Cell 2010; 141:483-496.

9 Kuenzel S, Till A, Winkler M, et al. The nucleotide-binding oligomerization domain-like receptor NLRC5 is involved in IFN-dependent antiviral 
immune responses. J Immunol 2010; 184:1990-2000.

10 Neerincx A, Lautz K, Menning M, et al. A role for the human nucleotidebinding domain, leucine-rich repeatcontaining family member NLRC5 in antiviral responses. J Biol Chem 2010; 285:26223-26232.

11 Kumar H, Pandey S, Zou J, et al.
NLRC5 deficiency does not influence cytokine induction by virus and bacteria infections. J Immunol 2011; 186:994-1000.

12 Davis BK, Roberts RA, Huang MT, et al. Cutting edge: NLRC5-dependent activation of the inflammasome. $J$ Immunol 2011; 186:1333-1337.

13 Kofoed EM, Vance RE. Innate im- mune recognition of bacterial ligands by NAIPs determines inflammasome specificity. Nature 2011; 477:592-595.

14 Zhao Y, Yang J, Shi J, et al. The NLRC4 inflammasome receptors for bacterial flagellin and type III secretion apparatus. Nature 2011; 477:596-600. 